\title{
Energy Migration Study of Random Immobile Anthracene Derivatives by Time Resolved Fluorescence Anisotropy Decays
}

Jian Yang, Robert S. Roller, Mitchell A. Winnik*

Department of Chemistry, University of Toronto, Lash Miller Chemical Laboratories, 80 St. George Street, Toronto, Ontario, Canada M5S 3H6

\section{Supporting Information}

Table S1. Fitting results of the total fluorescence decays $\left(I_{\text {sum }}\right)$ for the samples of 2-An-M in PMMA and the PI-2An-PMMA samples ${ }^{\text {a }}$

\begin{tabular}{|c|c|c|c|c|c|c|}
\hline Sample & $C_{\mathrm{An}}(\mathrm{mM})$ & $f_{1}{ }^{\mathrm{b}}$ & $\tau_{1}(\mathrm{~ns})$ & $f_{2}{ }^{\mathrm{b}}$ & $\tau_{2}(\mathrm{~ns})$ & $\left\langle\tau>^{\mathrm{c}}\right.$ \\
\hline \multirow{4}{*}{$\begin{array}{c}\text { 2-An-M in } \\
\text { PMMA }\end{array}$} & 1.2 & 0.42 & 2.4 & 0.58 & 4.3 & 3.5 \\
\cline { 2 - 7 } & 23.7 & 0.22 & 2.1 & 0.78 & 3.8 & 3.4 \\
\cline { 2 - 7 } & 48.9 & 0.28 & 2.2 & 0.72 & 3.9 & 3.4 \\
\cline { 2 - 7 } & 99.7 & 0.35 & 2.4 & 0.65 & 4.0 & 3.4 \\
\cline { 2 - 7 } & 125 & 0.39 & 2.4 & 0.61 & 4.0 & 3.4 \\
\hline \hline \multirow{3}{*}{ PI-2An-PMMA } & 170 & 0.43 & 2.1 & 0.57 & 4.0 & 3.2 \\
\cline { 2 - 7 } & 4.43 & 0.44 & 2.4 & 0.56 & 7.0 & 5.0 \\
\cline { 2 - 7 } & 16.5 & 0.38 & 2.2 & 0.62 & 7.3 & 5.4 \\
\hline
\end{tabular}

a Obtained by fitting $I_{\text {sum }}$ with double exponential decay function $I_{\text {sum }}(t)=A_{1} \exp \left(-t / \tau_{1}\right)^{+}$ $A_{2} \exp \left(-t / \tau_{2}\right)$. The $A_{i}$ and $\tau_{i}(i=1,2)$ are the coefficient and lifetime in the double exponential function.

b The fraction, $f_{i}$, of $\tau_{i}(i=1,2)$ was calculated based on $f=A_{2} \tau_{2} /\left(A_{1} \tau_{1}+A_{2} \tau_{2}\right)$.

c Obtained by eq 3 in the paper.

\section{Determination of Förster Radius of 2-An-M in PMMA.}

Energy transfer between two like or unlike chromophores can only occur if there is spectral overlap of the emission spectrum of the donor and the absorption spectrum of the acceptor. The emission spectrum of 2-An-M overlaps with its own absorption spectra (Figure S1), which makes 
energy migration possible if the concentration of 2-An-M is high enough in the matrix. The Förster radius, $R_{0}$, of 2-An-M in PMMA can be determined using the spectral overlap method. We briefly describe this method as follows.

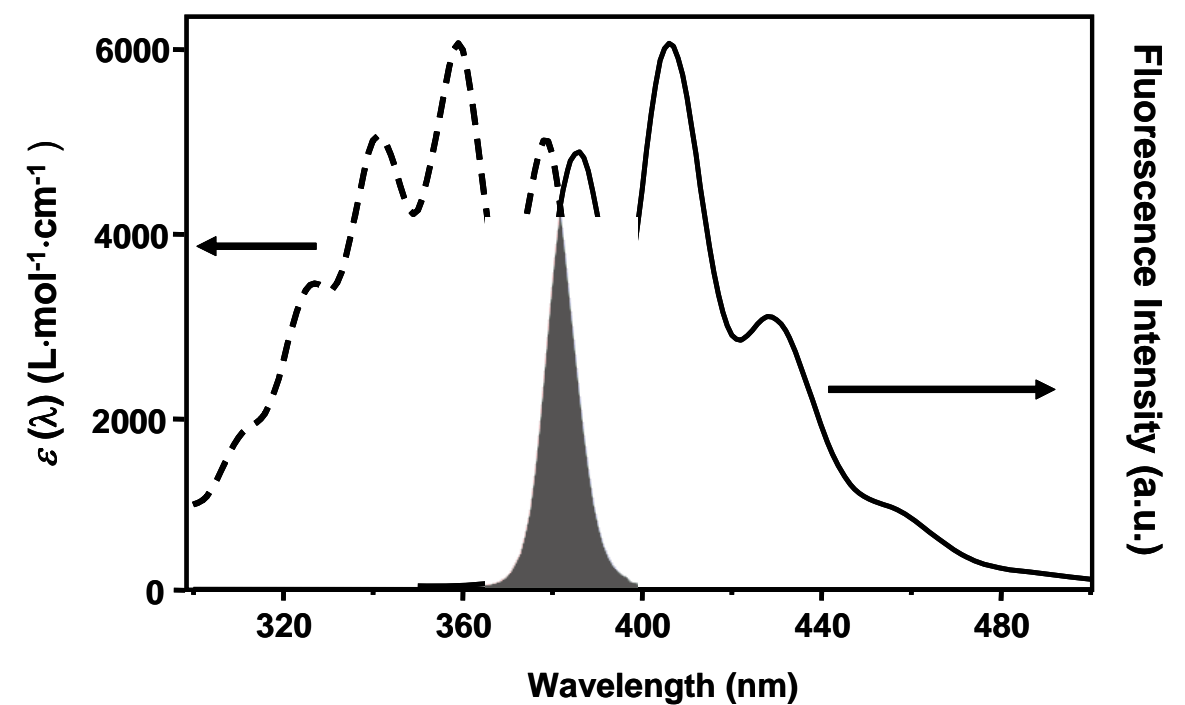

Figure S1 Spectral overlap (gray area) of 2-An-M in PMMA. Solid and dotted lines are the emission and absorption spectra respectively. The emission spectrum was measured by setting $\lambda_{\mathrm{ex}}=330 \mathrm{~nm}$.

The Förster radius for immobile dipoles is given by eq S1 with the assumption of $\langle|\kappa|\rangle^{2}=$ 0.476. The magnitude of each of the terms in this expression can be measured individually. ${ }^{\mathrm{s} 1}$

$$
R_{0}^{6}=0.476 \frac{9000(\ln 10) \Phi_{f}}{128 \pi^{5} N_{A V} n^{4}} \frac{\int_{0}^{\infty} F_{D}(\lambda) \varepsilon_{A}(\lambda) \lambda^{4} d \lambda}{\int_{0}^{\infty} F_{D}(\lambda) d \lambda}=0.252 \Phi_{f} J / N_{A V} n^{4}
$$

The corrected fluorescence spectrum $F_{D}(\lambda)$ was measured for 2-An-M dissolved in a PMMA matrix mounted on a quartz substrate. The spectrum $\varepsilon_{A}(\lambda)$ was determined by acquiring a series of absorption spectra for a series of PMMA films containing a constant concentration of 2-An-M (5.11 × $10^{-3} \mathrm{M}$ ) with thicknesses ranging from $70 \mu \mathrm{m}$ to $500 \mu \mathrm{m}$. Next, the extinction coefficient was determined from the slope of the Beer's Law plot of absorbance vs. thickness at the three wavelengths corresponding to the maxima of the three lowest energy vibronic transitions, and these values were 
used to scale the absorption spectrum to $\varepsilon_{\mathrm{A}}(\lambda)$. Correction of $F_{\mathrm{D}}(\lambda)$ for background fluorescence and $\varepsilon_{\mathrm{A}}(\lambda)$ for background absorbance originating from impurities in the PMMA matrix were carried out as described in Ref. S1.

The quantum yield determination was performed through area integration of the corrected fluorescence spectra of four samples of optical density varying from 0.01 to 0.04 in ethyl acetate solution. These were measured against the secondary reference standard quinine sulphate in $0.5 \mathrm{M}$ sulphuric acid. The quantum yield in ethyl acetate $\left(\Phi_{f}^{\text {EtAc }}\right)$ was taken to be the linear extrapolation of these four fluorescence quantum yields to zero optical density. The predicted quantum yield of fluorescence in PMMA $\left(\Phi_{f}^{\text {PMMA }}\right.$ ) was then calculated according to the equation

$$
\frac{\Phi_{f}^{\text {PMMA }}}{\Phi_{f}^{E t A c}}=\frac{n_{P M M A}^{2} \tau_{D}^{P M M A}}{n_{E t A c}^{2} \tau_{D}^{E t A c}}
$$

where $\tau_{\mathrm{D}}{ }^{\mathrm{X}}$ is the fluorescence lifetime of 2-An-M in medium $\mathrm{X}$ as obtained by time-correlated single photon timing. As well, $n_{\mathrm{X}}$ is the tabulated refractive index of $\mathrm{X}$ at $25^{\circ} \mathrm{C}$, i.e., 1.437 for ethyl acetate and 1.492 for PMMA. The latter value was also employed for $n$ in eq S2. The value of $\Phi_{f}^{\text {EtAc }}$ was determined to be 0.21 and, based on this value, we calculated a value of $\Phi_{f}^{\text {PMMA }}=0.24$. The Förster radius for energy migration for 2-An-M in PMMA, based on the results of the quantum yield and spectral overlap integral determinations, can thus be calculated from eq $\mathrm{S} 1$, and we found $R_{0}=1.8 \pm$ $0.1 \mathrm{~nm}$.

\section{REFERENCE}

${ }^{\mathrm{S} 1}$ Roller, R. The Determination of the Förster Critical Radius through Time-Dependent Fluorescence Decay Measurements and the Spectral Overlap Method. Master thesis, University of Toronto, 2004. 